DOE/ER/60588-1

\title{
THE IMPACT OF ENERGY RELATED POLLUTANTS ON CHROMOSOME STRUCTURE
}

\section{Final Performance Report}

Grant Period: May 1, 1987 - April 30, 1992

Randolph L. Rill

Principal Investigator

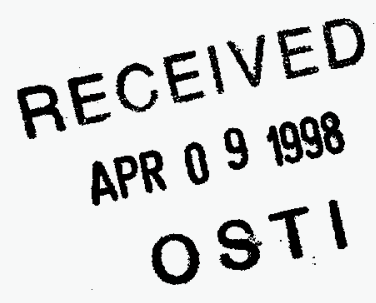

Institute of Molecular Biophysics

The Florida State University

Tallahassee, FL 32306

Report Date: 3/19/98

PREPARED FOR THE U.S. DEPARTMENT OF ENERGY UNDER GRANT NUMBER DE-FG05-87-ER60588

DISTRIBUTION OF THIS DOCUMENT IS UNIMIED

MASTER 


\section{DISCLAIMER}

This report was prepared as an account of work sponsored by an agency of the United States Government. Neither the United States Government nor any agency thereof, nor any of their employees, makes any warranty, express or implied, or assumes any legal liability or responsibility for the accuracy, completeness, or usefulness of any information, apparatus, product, or process disclosed, or represents that its use would not infringe privately owned rights. Reference herein to any specific commercial product, process, or service by trade name, trademark, manufacturer, or otherwise does not necessarily constitute or imply its endorsement, recommendation, or favoring by the United States Government or any agency thereof. The views and opinions of authors expressed herein do not necessarily state or reflect those of the United States Government or any agency thereof. 


\section{DISCLAIMER}

Portions of this document may be illegible electronic image products. Images are produced from the best available original document. 


\section{Summary}

This project addressed the sequence selectivities of DNA binding by intercalating agents. Methods analogous to chemical DNA sequencing were developed to quantitatively investigate sequence selectivities of DNA binding of several DNA intercalators including benzo(a)pyrene diol epoxides, ethidium, copper-phenanthroline complexes, and the anticancer drug actinomycin D. Computer programs were developed to extract sequence selectivities from large data sets. A photoaffinity analog approach was validated for determining the sequence selectivities of ethidium and actinomycin D. Several 'non-traditional' binding sites were identified for each ligand examined. Actinomycin D was shown to bind single stranded DNA, as well as double stranded DNA, with high affinity and sequence selectivity.

All of the compounds studied were intercalators, but they differ significantly in side chain complexity. The degree of sequence preference for ligand binding increased with increasing complexity, a result that is not surprising, but lends credence to the methods used. Despite the obvious influences of the side chain, several common themes were noted that indicate fundamental preferences for binding by intercalation. Several general conclusions can be drawn from the collective analyses.

1. There are no "simple" rules. Classic descriptions in terms of GC, AT, or "pyrimidine-purine step" specificities are often misleading.

2. There are strong influences of both nearest-neighbors of a base on reactivity.

3. Next nearest-neighbors also significantly influence reactivities.

4. YRY and YGG sequences are generally good binding sites for intercalators, particularly if the 5'-pyrimidine is T. Oligopurine/oligopyrimidine tracts are generally poor sites of intercalator binding, but are not totally avoided.

These conclusions conform only in part to currently accepted concepts of intercalator binding. A strong dependence of reactivity on one of the bases flanking the modified base is expected to be related to the energetics of base unstacking and the stereo/electronic "fit" of the ligand within the intercalation site. These effects can be investigated by standard molecular modeling techniques. The influences of next-nearest-neigbors of the intercalation site are hard to comprehend in these terms for simple intercalators lacking extended side chain substituents such as ethidium and BPDEs, and are most likely to reflect subtle influences of the local, sequence-dependent DNA conformation in the ground (unbound) or perturbed (bound) states. Understanding of these influences is best approached by experimentally determining the structures of particular sequences in model duplexes alone and complexed with ligand.

\section{Detailed Progress Report.}

\section{Overview. Detection of non-random binding of intercalating ligands.}

Intercalators are important DNA ligands. Some act as potent carcinogens, while others are well accepted anticancer drugs. This project examined the degrees to which various intercalating agents exhibited sequence preferences in DNA binding. Knowledge of sequence preferences of intercalators could assist in understanding mechanisms of carcinogenesis by compounds such as metabolites of polycyclic aromatic hydrocarbons and amines. They may also assist in design of sequence-specific anticancer drugs.

The intercalators studied included two expected to exhibit significant specificity (7-azido-actinomycin D, azido-ActD, and bis-(1,10-phenanthroline) $\mathrm{Cu}(\mathrm{I}),(\mathbf{P h e n})_{2} \mathrm{Cu}(\mathrm{I})$ ), one expected to show little specificity (8-azido-ethidium, azido-Et), and the $(+)$ - and (-)-isomers of benzo(a)pyrene - 7,8 - diol- 9,10 - epoxide (BPDE). All ligands interact non-randomly with DNA and display different degrees of selectivity of reactive base type and the sequence context.

The experimental approach used techniques analogous to DNA sequencing to "map" sites of ligand binding, and quantitate the binding. This approach depends on the fact that the above chemicals either spontaneously cleave DNA, or form adducts that promote DNA chain cleavage by 
hot piperidine, as in chemical DNA sequencing. (Phen) ${ }_{2} \mathrm{Cu}(\mathrm{I})$ spontaneously cleaves DNA cleavage via an active oxygen species. Azido-ActD and azido-Et are photoaffinity analogs of the parent compounds which covalently couple to DNA at the binding site when photolyzed by visible light. BPDE contains a ring epoxide that readily undergoes nucleophilic addition. The latter compounds were all found to produce alkali-labile adducts of all bases, but preferentially at guanines.

\section{Identification of Preferred Sequences of Ligand Binding.}

Several programs were written to identify and quantitatively compare sequences preferred by individual ligands, at the individual base level, from band intensities measured on autoradiograms. Data on sequences of at least 1200 bases were obtained for each of the ligands listed above. These data sets were sufficient to identify many major trends in binding preferences.

With the exception of $(\mathrm{Phen})_{2} \mathrm{Cu}(\mathrm{I})$, we have found that the above ligands exhibit reactivities towards bases in the order $\mathrm{G}>\mathrm{A} \geq \mathrm{C}>\mathrm{T}$. We used several approaches to provide a quantitative measure of preferences. A plot of the number distribution of band intensities provides a visual representation of the range of reactivities of a particular base type and is useful for comparing the ranges of reactivities of different ligands. A quantitative measure of the relative reactivities of ligands with a particular base is provided by comparing the ratios of the band intensities of the "high" and "low" reactivity groups for the ligands. The upper and lower 10 percentile ranges were chosen for comparison. For example, the most and least reactive guanines, defined in terms of the upper and lower 10 percentiles, differed by a factor of $\approx 17$ in reactivity towards both BPDE isomers. The most reactive guanines were about $3-3.2$ times as reactive as the average guanine. By comparison, the guanines most and least reactive with azido-ActD differed by a factor of 40 , while these most and least reactive with azido-Et differed by a factor of 5. If formation of the non-covalent complexes were rate limiting in each case, the band intensities could be interpreted in terms of site populations--hence non-covalent association constants--and in which case these differences in reactivity correspond to approximately $2.2,1.7$, and 0.97 $\mathrm{Kcal} / \mathrm{mole}$ differences in standard free energy of non-covalent between the most and least reactive sites of binding of azido-ActD, anti-(-)-BPDE, and azido-Et, respectively. (Non-covalent binding appears not to be the rate limiting step in BPDE adduct formation, however.)

The sequence preferences of ligands were examined at the levels of all doublets, triplets and quartets including the target base (e.g., G). Sequences surrounding G's that were highly preferred were identified using two criteria: the propensity for occurrence of the sequence in the highly preferred group, and the band intensity of the target guanine of the sequence averaged over all occurrences of the sequence. These two criteria are useful because they measure different aspects of the sequence preferences. The average band intensity for cleavage at a guanine in a particular sequence is a quantitative measure of the degree of preference for reaction at that sequence, relative to other sequences. The propensity for occurrence of a specific sequence in the preferred group is a measure of the sufficiency of that sequence for determining a high reactivity.

We found that at least triplet sequences are required to define the sequence preferences of all ligands studied. The traditional references to GC, AT, of "pyrimidine-purine step" preferences of various ligands are relatively meaningless or too simplistic. In addition, for all ligands we identified several "surprise" sequences that are strongly preferred; examples are: TGGG for azido-ActD, TGG for azido-Et, and AGG for BPDE. In many cases there are clearly influences of the next-nearest neighbors on the reactivity of a base, hence at least quartets or pentamers are required to fully characterize the preferences. These effects largely cannot be explained in terms of simple stereo/electronic factors or current knowledge of DNA conformation.

\footnotetext{
The Sequence Preferences of Piperidine-Labile Adduct Formation by Anti-BPDE Isomers.

Data were obtained on piperidine cleavage of adducts of anti-(+)- and anti-(-)-BPDE isomers. Both isomers most frequently with guanines, and these reactions were non-random. The two BPDE isomers were similar in selectivity, and were about half as selective as azido-actinomycin $\mathrm{D}$, but about three times as selective as azido-ethidium.
} 
The 5'-guanines in GG doublets not preceded by a guanine were most reactive with anti-(-)-BPDE on average. The second and successive G's in oligo-G tracts were not unusually reactive. This result contrasts with that of Boles and Hogan, who noted strong and approximately equal cleavage at all G's in guanine tracts upon photolysis of DNA modified by racemic anti-BPDE with $355 \mathrm{~nm}$ laser light. Since racemic anti-BPDE predominantly forms piperidine-stable guanine $\mathrm{N}^{2}$ adducts, this difference between the two results may reflect differences in the sequence requirements for BPDE addition at alternative sites.

Guanines flanked on the 5 -side by $T$ were also generally more reactive than average with anti-(-)-BPDE, with the triplet preferences being in the order TGG $\geq$ TGT $>$ TGA $\geq$ TGC. By contrast, anti-(+)-BPDE strongly preferred AGG and CGG triplets, but TGG triplets were only slightly preferred, and other TGN triplets exhibited average reactivity. More detailed examination of anti-(-)-BPDE preferences showed that bases 5'- and $3^{\prime}$-flanking the preferred triplets affected reactivities. We have found that the sequences preferences of all chemicals examined are expressed better at the level of triplets, rather than doublets. A more comprehensive analysis of the reactivities of guanines with anti-(-)-BPDE indicated the following trends. (Abbreviations are: $R$ $=$ purine, $\mathbf{Y}=$ pyrimidine, the target $\mathbf{G}$ is in bold.)

- $\quad$ RGR sequences are less reactive than average, excepting AGG.

- $\quad$ AGG sequences are highly reactive unless flanked by a G.

- $\quad$ Of less reactive RGR sequences, RGRY sequences are the least reactive.

- RGY sequences are usually less reactive than average; none are more reactive than average.

- $\quad$ YGR sequences are generally more reactive than average, but with several exceptions.

- $\quad$ A 5'-flanking purine (RYGR) generally enhances reactivity.

- $\quad$ A 5'-flanking pyrimidine (YYGR) generally enhances reactivity

- $\quad$ Effects of 3'-flanking bases are variable.

- YGY sequences are generally slightly more reactive than average except

- $\quad$ YYGT and YGTY (excepting CGTT) are much above average.

- $\quad$ CGCT reactivities are below average.

Sequence preferences for anti-(-)-BPDE addition at other bases were also investigated. The most reactive adenines occurred in the following quartets $(\mathrm{N}=$ any base): (T/G) $\underline{A} G T, C G \underline{A}(A / T)$, (T/C)ACC, CCAN, and NAGG. The most reactive cytosines occurred in the following quartets: (A/T)TCGN, (A/C) $\underline{C}(\mathrm{G} / \mathrm{C}), \mathrm{GCCT}, \mathrm{ACC}(\mathrm{C}>\mathrm{A})$, and CCEC. The quartets $(\mathrm{A} / \mathrm{T}) \mathrm{TCG}(\mathrm{A} / \mathrm{T} / \mathrm{C})$ are not preferred sites of guanine addition. The remaining sequences are related to the high preference for G's in G doublets or oligo-G tracts. The preferred reactivity of the $A C C(G / C)$ quartets is noteworthy because high reactivity of the $A$ in $(T / C)$ ACC sequences was also observed, although the last G in GGT sequences is not highly preferred. Studies with azido-actinomycin D and azido-ethidium have also indicated that there is something unique about TGGT and TGGGT sequences.

\section{Sequence Preferences of DNA Binding by Azido-Ethidium.}

We used 8-azido-ethidium (azido-Et), in which the 8-amino group of the parent ethidium is replaced by a photo-labile azide moiety, as a photoaffinity probe of the preferred sites of ethidium binding. 8-Azido ethidium binds to DNA in a manner virtually indistinguishable from the parent ethidium in terms of the binding constant and neighbor exclusion parameter, and certain spectroscopic properties. Azido-ethidium and other aromatic azido compounds are photo-labile in visible light and form base adducts labile to hot piperidine, allowing mapping of adduct sites by sequencing techniques.

Azido-Et treated DNA was cleaved at all bases, with yields of labile adducts being in the order $\mathrm{G}>\mathrm{C} \geq \mathrm{T}>\mathrm{A}$. The degree of sequence preference observed with azido-Et was less than that of anti-BPDE, (Phen) ${ }_{2} \mathrm{Cu}^{+}$, or azido-ActD. Nonetheless, definite preferences were noted, and the least and most preferred sequences differed in reactivity by factors of 4-5. This factor of 5 could correspond to a significant difference in $\Delta \mathrm{Go}$ binding of about $1 \mathrm{Kcal} / \mathrm{mole}$.

As observed with anti-BPDE isomers, the most reactive $G$ 's on average occurred in GG doublets. TG, $\mathrm{G} \underline{\mathrm{G}}(\mathrm{T}>\mathrm{G}>\mathrm{C})$, and $\mathrm{C} \underline{G}$ were the only triplets with a central $\mathrm{G}$ that was 
significantly more reactive than average. Bases next-nearest the reactive guanine exerted significant effects. The most preferred quartets are represented by: YGG $Y$, TGGG, GTGG, ACGC, TCGT and TGGT, GGT(A,G>T), GGG(G>A,T), GGCA, CGTA, TGAG. The strongest pentamer preferences suggested are therefore YGGYA, TGGGG, GTGGT, and TCGTA. Similar analyses were performed for cleavages at other bases. Considered together, these data suggest that YGG and YRY sequences are generally strong ethidium binding sites. Comparison of the reactivities of the three bases in these triplets suggests that intercalation occurs in the pyrimidine-3',5'-purine step of YRY triplets, as expected. By contrast, the high reactivities of both G's in YGGY sequences, particularly TGGT, suggests that intercalation occurs between the two G's.

\section{Sequence Preferences of DNA Binding by Azido-Actinomycin D.}

The sequence preferences of 7-azido-actinomycin $D$ were examined as a test of our procedures because this compound is one of the most "sequence-specific" DNA binding drugs known, and the binding has been well characterized by other means. Actinomycin D (ActD) is an antitumor antibiotic that appears to act in vivo by inhibiting RNA polymerase. The mechanism of non-covalent DNA binding of ActD has attracted much attention because of its strong preference for 5'-GpC-3' sequences in dsDNA. Binding at GC steps involves intercalation of the tricyclic aromatic phenoxazone ring into a GC step, with the two equivalent cyclic pentapeptide lactone substituents lying in the minor groove and making hydrogen bond contacts with the 2-amino groups of the nearest neighbor guanines.

Binding is also influenced by next-nearest neighboring bases. We examined this higher order specificity using 7-azido-actinomycin $\mathrm{D}$ as a photoaffinity probe as described for azido-ethidium. GC doublets were strongly preferred addition sites, as expected, but only if the 5'-flanking base was a pyrimidine and the 3'-flanking base was not cytosine. In addition we observed a strong preference for binding at a GG doublet in 5'-TGGG-3', and exceptionally strong reaction with TGGGT.

Strong non-covalent binding of ActD to the non-traditional sequences TGGT and TGGGT was subsequently confirmed by spectroscopic titration studies of ActD binding to model oligonucleotide duplexes. During the course of these studies we noted strong ActD binding to single stranded DNA oligomers with ACCA, ACCCA and ACCCCA central sequences. A primer extension assay was used to show that ActD binds with high sequence selectivity to single stranded DNA and binding is sufficiently strong to inhibit a number of DNA polymerases, including HIV Reverse Transcriptase.

\section{DNA Binding and Cleavage by Bis-(1,10-phenanthroline) $\mathrm{Cu}(\mathrm{I})$.}

The sequence preferences of DNA cleavage by (Phen $)_{2} \mathrm{Cu}+$ were determined by mapping sites on $>2000 \mathrm{bp}$ in DNA restriction fragments. We found that ( $\mathrm{Phen})_{2} \mathrm{Cu}+$ has a predominant preference for TAT triplets, and a moderate preference for TGT triplets; and also a strong preference for TAAT, TAGPy, and CAGT quartets. Oligopurine and oligopyrimidine tracts are poorly reactive. We have also shown that (Phen $)_{2} \mathrm{Cu}+$ binds in the minor groove at preferred sequences. These reaction specificities are unusual for a potentially intercalating DNA ligand, and can be explained mainly in terms of a requirement for binding at the minor groove side of a pyrimidine-3',5'-purine step and the stereoelectronic effects of 2-amino groups of guanine, which inhibit minor groove binding.

To test the influence of the guanine 2-amino group on specificity we synthesized the undecamers of the general formula: CCCT(PyPuPy)CCCC, where PyPuPy = TAT, CAT, TGT, TIT $(\mathrm{I}=$ inosine $)$ and TAC; and their complements: GGGA(PuPyPu)GGGG, where PuPyPu = ATA, GTA, ITA, ACA, ATG, and ATI. Minihelices of complementary oligonucleotides. containing TAT, TGT and TIT were cleaved strongly at the central purine and weakly at other positions, as expected from results obtained with long DNA fragments, demonstrating that local helix geometry is sufficient to define the cleavage specificity. Substitution of I for $G$ in the oligomers containing CAT:ITA and TIT:ACA significantly enhanced cleavage at the central purine in PyPuPy sequences, confirming that a guanine 2-amino group in the minor groove inhibits 
binding. The oligomers containing central TAC:ATG and TAC:ATI sequences, however, exhibited similar reactivity that was reduced relative to TAT, indicating that more subtle factors influence the base preference at the third position in PyPuPy triplets.

We developed techniques to perform spectral and viscometric titrations of DNA with (Phen) $)_{2} \mathrm{Cu}+$ under oxygen-free conditions (required to prevent oxidation of $\mathrm{Cu}^{+}$to $\mathrm{Cu}++$, and examined (Phen $)_{2} \mathrm{Cu}+$ binding to random sequence DNA. The spectral properties of the (Phen) ${ }_{2} \mathrm{Cu}+/ \mathrm{DNA}$ complex and the effect of (Phen) ${ }_{2} \mathrm{Cu}+$ on the viscosity of DNA solutions are consistent with an intercalation binding mechanism. The spectrum of the complex exhibited strong hypochromism and a strong induced circular dichroism spectrum consistent with exciton coupling, and addition of (Phen) ${ }_{2} \mathrm{Cu}+$ to DNA caused a large increase in DNA solution viscosity. The binding isotherm was complex, suggesting positive cooperativity, and was dependent on the concentration of excess phenanthroline. Excess phenanthroline also had subtle effects on the absorption properties of the (Phen) ${ }_{2} \mathrm{Cu}+$ :DNA complex. (Phenanthroline is required in excess to assure that ( $\mathrm{Phen})_{2} \mathrm{Cu}+$ is the predominant $\mathrm{Cu}+$ species.) In all cases binding was well described by $\mathrm{McGhee-Von} \mathrm{Hippel} \mathrm{theory} \mathrm{with} \mathrm{a} \mathrm{neighbor} \mathrm{exclusion} \mathrm{parameter} n \approx 2$, as found for many intercalators, and a positive cooperativity parameter of 3.0 to 4.6 , depending on the excess phenanthroline concentration. Binding measurements were also performed with the redox-inactive bis-(2,9-dimethyl-1,10-phenanthroline) $\mathrm{Cu}(\mathrm{I})$ complex, and with phenanthroline alone. No DNA binding of these two compounds could be detected by spectroscopic or viscosity criteria, although phenanthroline appeared to be a competitive inhibitor of ( $\mathrm{Phen})_{2} \mathrm{Cu}+$ binding.

Mono-phenanthroline: $\mathrm{Cu}(\mathrm{I})$ complex appeared to bind DNA by intercalation, however, as indicated by the large increase in DNA solution viscosity when titrated with 1:1

$\mathrm{Cu}(\mathrm{I})$ :phenanthroline.

During the course of the above studies we noted that the characteristic visible spectrum of (Phen) ${ }_{2} \mathrm{Cu}^{+}$was not obtained in the presence of the reducing agent mercaptopropionic acid (MPA), but was formed in the presence of ascorbate, an alternative reducing agent. Additional visible and NMR spectroscopic studies showed that the (Phen) ${ }_{2} \mathrm{Cu}^{+}$complex was disrupted by MPA, perhaps due to formation of an (MPA)(Phen)Cu(I) complex. This finding raised the possibility that the cleavage preferences observed previously were due to this uncharacterized species, not (Phen) ${ }_{2} \mathrm{Cu}+$. We subsequently showed, however, that the preference for TAT was still observed when ascorbate was substituted for MPA as the reducing agent, and that the DNA cleavage reaction proceeded much faster with ascorbate as reductant. Excess phenanthroline accelerated cleavage in the presence of MPA, but decreased cleavage in the presence of ascorbate. We conclude that the (Phen) ${ }_{2} \mathrm{Cu}+$ complex is the major DNA cleaving agent even in the presence of MPA, but there is competition between MPA and phenanthroline for $\mathrm{Cu}^{+}$. The decrease in DNA cleavage by phenanthroline when ascorbate was the reductant is consistent with the inhibitory effect of phenanthroline on (Phen) ${ }_{2} \mathrm{Cu}+$ binding noted above. In addition, when the $\mathrm{Cu}$ :Phen ratio was reduced to 1:1 we observed a strong preference for cleavage at the central CG of a 5'-CCGGG-3' sequence, and very reduced cleavage at a nearby $\mathrm{TAT}$, which was highly preferred at a Cu:Phen ratio of 1:2. This result is consistent with observations that $\mathrm{N}$-alkyl phenanthrolinium ions prefer GC-rich DNA over AT-rich DNA, and directly demonstrates the strong influence of the second phenanthroline ring in directing the sequence preference of the ( $\mathrm{Phen})_{2} \mathrm{Cu}+$ complex to TA rather than CG steps.

All of the spectroscopic and oligonucleotide cleavage data obtained are consistent with a model we have proposed for (Phen $)_{2} \mathrm{Cu}+$ binding to preferred TAT sequences in which one phenanthroline intercalates in the T-3',5'-A step from the minor groove side, and the second phenanthroline ring of the complex lies in the minor groove, nearly parallel with the helix axis and approximately in the positions occupied by the guanine $\mathrm{N}^{2}$-amino group in a C-3', $5^{\prime}-\mathrm{G}$ step \{\} . This positioning is consistent with the low degree of DNA cleavage observed at CG steps and CAT sequences, and the relative rates of cleavage at different nucleotide sugars in the binding site \{\} . 


\section{Publications Resulting From Grant Period 5/1/87-4/30/92}

Veal*, J. and Rill, R. L., "The Sequence Specificity of DNA Cleavage by (Phen $)_{2} \mathrm{Cu}+$ ", Biochemistry 27, 1822-1827 (1988).

Veal*, J. M. and Rill, R. L., "Sequence Specificity of DNA Cleavage by Bis-(1,10)phenanthroline $\mathrm{Cu}(\mathrm{I})$ : Effects of Single Base Pair Transitions on the Cleavage of Preferred Pyrimidine-Purine-Pyrimidine Triplets", Biochemistry 28, 3243-3250 (1989)

Rill, R. L., Marsch*, G., and Graves, D., "The Sequence Specificity of Actinomycin D Binding to DNA", J. Biomol. Str.Dynam.7, 591-606 (1989)

Rill, R. L., and Marsch*, G., "Sequence Preferences of Covalent Binding DNA Binding by Anti(+)- and Anti-(-)-Benzo(a)pyrene-7.8-diol-9,10-epoxide", Biochemistry 29, 6050-6058 (1990).

Veal*, J.M. and Rill, R.L., "The Non-Covalent DNA Binding of Bis-(1,10-phenanthroline)Cu(I) and Related Compounds", Biochemistry 30, 1132-1140 (1991).

Veal*, J.M., Merchant*, K., Chen, Q., and Rill, R.L., "The Influence of Reducing Agent and Phenanthroline Concentration on DNA Cleavage by 1,10-Phenanthroline:Copper", Nucleic Acids Res. 19, 3383-3388 (1991).

Bailey,S.A., Graves, D.E., Rill, R.L., and Marsch*,G.A., "Influence of DNA Base Sequence on the Binding Energetics of Actinomycin D", Biochemistry, 32, 5881-5887 (1993).

Bailey,S.A., Graves, D.E., and Rill, R.L., "Binding of Actinomycin D to the T(G) $T$ T Motif of Double Stranded DNA: Determination of the Guanine Requirement in Nonclassical, NonGpC Binding Sites", Biochemistry 33, 11493-11500 (1994).

Marsch*, G.A., Graves, D.E. and Rill, R.L., "Photoaffinity Approaches to Determining the Sequence Selectivities of DNA-Small Molecule Interactions: Actinomycin D and Ethidium", Nucleic Acids Research 23, 1252-1259 (1995).

Rill, R.L., and Hecker*, K.H. , "Sequence-specific Actinomycin D Binding to Single Stranded DNA Inhibits HIV Reverse Transcriptase and Other Polymerases ", Biochemistry35, 3525-3533 (1996).

\section{Predoctoral Associates Trained With Support From This Grant.}

Carolyn Waldron, M.S. in Biology, 1988

Teresa Strzelecka, Ph.D. in Molecular Biophysics, 1988

James M. Veal, Ph.D. in Molecular Biophysics, 1989

Glenn Marsch, Ph.D. in Molecular Biophysics, 1990

Karl Hecker, Ph.D. in Chemistry, 1993 